\title{
Bayesian Group Lasso for Nonparametric Varying-Coefficient Models With Application to Functional Genome-Wide Association Studies
}

Jiahan Li and Zhong Wang and Runze Li and Rongling Wu

Supplementary Material

Convergence diagnostics and summary of parameter estimates.

(doi: COMPLETED BY THE TYPESETTER). 
TABLE 1

Average estimates, standard errors, and mean squared errors (MSEs) in the simulated example using Bayesian group lasso when $n=600$.

\begin{tabular}{lccccccccccccc}
\hline \multicolumn{1}{l}{ Parameter } & \multicolumn{1}{c}{ Estimated Legendre Coefficients } & \multicolumn{3}{c}{ Standard Errors } \\
\hline$\rho_{G}=0.1, n=600, \sigma^{2}=16$ & & & & & & & & & \\
$\mathbf{b}_{1}$ & 1.03 & 0.89 & -2.04 & -0.01 & 0.10 & 0.16 & 0.18 & 0.20 & 0.01 & 0.03 & 0.03 & 0.04 \\
$\mathbf{b}_{2}$ & 1.18 & -0.24 & 0.76 & -4.74 & 0.10 & 0.15 & 0.21 & 0.18 & 0.01 & 0.02 & 0.05 & 0.03 \\
$\mathbf{b}_{3}$ & 1.41 & 0.00 & -0.03 & 0.00 & 0.09 & 0.16 & 0.17 & 0.21 & 0.01 & 0.03 & 0.03 & 0.05 \\
$\mathbf{c}_{3}$ & 1.61 & -2.21 & 4.83 & 1.36 & 0.13 & 0.21 & 0.30 & 0.30 & 0.03 & 0.05 & 0.09 & 0.09 \\
$\mathbf{c}_{4}$ & 1.23 & 1.20 & 1.94 & 1.45 & 0.13 & 0.21 & 0.24 & 0.30 & 0.07 & 0.06 & 0.06 & 0.09 \\
$\mathbf{c}_{5}$ & 1.43 & -1.29 & 0.03 & -0.05 & 0.11 & 0.24 & 0.26 & 0.31 & 0.04 & 0.06 & 0.07 & 0.10 \\
$\rho_{G}=0.1, n$ & $600, \sigma^{2}=9$ & & & & & & & & & & \\
$\mathbf{b}_{1}$ & 1.03 & 0.89 & -2.06 & 0.02 & 0.06 & 0.12 & 0.16 & 0.15 & 0.00 & 0.02 & 0.03 & 0.02 \\
$\mathbf{b}_{2}$ & 1.18 & -0.23 & 0.72 & -4.73 & 0.07 & 0.11 & 0.15 & 0.18 & 0.00 & 0.01 & 0.02 & 0.03 \\
$\mathbf{b}_{3}$ & 1.40 & 0.01 & 0.00 & 0.01 & 0.07 & 0.12 & 0.17 & 0.16 & 0.00 & 0.01 & 0.03 & 0.03 \\
$\mathbf{c}_{3}$ & 1.55 & -2.14 & 4.90 & 1.34 & 0.10 & 0.18 & 0.20 & 0.22 & 0.01 & 0.03 & 0.04 & 0.05 \\
$\mathbf{c}_{4}$ & 1.07 & 1.27 & 1.91 & 1.45 & 0.09 & 0.16 & 0.17 & 0.23 & 0.01 & 0.03 & 0.03 & 0.05 \\
$\mathbf{c}_{5}$ & 1.32 & -1.25 & 0.02 & -0.04 & 0.09 & 0.15 & 0.19 & 0.18 & 0.01 & 0.02 & 0.04 & 0.04 \\
$\rho_{G}=0.1, n$ & $600, \sigma^{2}=4$ & & & & & & & & & & \\
$\mathbf{b}_{1}$ & 1.05 & 0.90 & -2.04 & -0.01 & 0.04 & 0.09 & 0.08 & 0.11 & 0.00 & 0.01 & 0.01 & 0.01 \\
$\mathbf{b}_{2}$ & 1.17 & -0.22 & 0.74 & -4.72 & 0.04 & 0.08 & 0.08 & 0.11 & 0.00 & 0.01 & 0.01 & 0.01 \\
$\mathbf{b}_{3}$ & 1.40 & -0.01 & 0.00 & 0.00 & 0.05 & 0.07 & 0.09 & 0.11 & 0.00 & 0.01 & 0.01 & 0.01 \\
$\mathbf{c}_{3}$ & 1.52 & -2.14 & 4.82 & 1.34 & 0.06 & 0.11 & 0.16 & 0.14 & 0.00 & 0.01 & 0.02 & 0.03 \\
$\mathbf{c}_{4}$ & 1.03 & 1.31 & 1.91 & 1.46 & 0.06 & 0.11 & 0.13 & 0.13 & 0.00 & 0.01 & 0.02 & 0.02 \\
$\mathbf{c}_{5}$ & 1.28 & -1.23 & 0.01 & -0.02 & 0.07 & 0.09 & 0.13 & 0.14 & 0.01 & 0.01 & 0.02 & 0.02 \\
\hline
\end{tabular}


TABLE 2

Average estimates, standard errors, and mean squared errors (MSEs) in the simulated example using Bayesian group lasso when $n=800$.

\begin{tabular}{lccccccccccccc}
\hline Parameter & \multicolumn{1}{c}{ Estimated Legendre Coefficients } & \multicolumn{3}{c}{ Standard Errors } \\
\hline$\rho_{G}=0.1, n=800, \sigma^{2}=16$ & & & & & & & & & \\
$\mathbf{b}_{1}$ & 1.04 & 0.86 & -2.06 & 0.01 & 0.08 & 0.13 & 0.17 & 0.17 & 0.01 & 0.02 & 0.03 & 0.03 \\
$\mathbf{b}_{2}$ & 1.16 & -0.21 & 0.74 & -4.70 & 0.08 & 0.12 & 0.16 & 0.16 & 0.01 & 0.01 & 0.03 & 0.03 \\
$\mathbf{b}_{3}$ & 1.40 & 0.00 & 0.03 & 0.02 & 0.08 & 0.14 & 0.16 & 0.21 & 0.01 & 0.02 & 0.03 & 0.04 \\
$\mathbf{c}_{3}$ & 1.58 & -2.18 & 4.85 & 1.31 & 0.12 & 0.17 & 0.21 & 0.25 & 0.02 & 0.03 & 0.04 & 0.07 \\
$\mathbf{c}_{4}$ & 1.08 & 1.26 & 1.94 & 1.40 & 0.10 & 0.16 & 0.24 & 0.24 & 0.02 & 0.03 & 0.06 & 0.07 \\
$\mathbf{c}_{5}$ & 1.33 & -1.26 & 0.04 & -0.07 & 0.11 & 0.17 & 0.23 & 0.29 & 0.02 & 0.03 & 0.05 & 0.09 \\
$\rho_{G}=0.1, n$ & $800, \sigma^{2}=9$ & & & & & & & & & & \\
$\mathbf{b}_{1}$ & 1.04 & 0.88 & -2.05 & 0.00 & 0.05 & 0.10 & 0.11 & 0.14 & 0.00 & 0.01 & 0.01 & 0.02 \\
$\mathbf{b}_{2}$ & 1.18 & -0.24 & 0.75 & -4.72 & 0.06 & 0.11 & 0.13 & 0.14 & 0.00 & 0.01 & 0.02 & 0.02 \\
$\mathbf{b}_{3}$ & 1.40 & 0.01 & -0.01 & 0.01 & 0.06 & 0.08 & 0.12 & 0.14 & 0.00 & 0.01 & 0.01 & 0.02 \\
$\mathbf{c}_{3}$ & 1.53 & -2.15 & 4.83 & 1.34 & 0.09 & 0.12 & 0.16 & 0.18 & 0.01 & 0.02 & 0.03 & 0.04 \\
$\mathbf{c}_{4}$ & 1.04 & 1.29 & 1.95 & 1.41 & 0.08 & 0.12 & 0.15 & 0.18 & 0.01 & 0.02 & 0.02 & 0.04 \\
$\mathbf{c}_{5}$ & 1.30 & -1.26 & 0.02 & -0.03 & 0.08 & 0.14 & 0.18 & 0.19 & 0.01 & 0.02 & 0.03 & 0.04 \\
$\rho_{G}=0.1, n$ & $800, \sigma^{2}=4$ & & & & & & & & & & \\
$\mathbf{b}_{1}$ & 1.04 & 0.88 & -2.05 & 0.00 & 0.04 & 0.06 & 0.08 & 0.09 & 0.00 & 0.00 & 0.01 & 0.01 \\
$\mathbf{b}_{2}$ & 1.17 & -0.22 & 0.74 & -4.70 & 0.04 & 0.07 & 0.08 & 0.10 & 0.00 & 0.01 & 0.01 & 0.01 \\
$\mathbf{b}_{3}$ & 1.40 & 0.00 & 0.00 & 0.00 & 0.04 & 0.06 & 0.07 & 0.11 & 0.00 & 0.00 & 0.00 & 0.01 \\
$\mathbf{c}_{3}$ & 1.51 & -2.12 & 4.82 & 1.39 & 0.05 & 0.09 & 0.11 & 0.12 & 0.00 & 0.01 & 0.01 & 0.02 \\
$\mathbf{c}_{4}$ & 1.02 & 1.29 & 1.91 & 1.46 & 0.05 & 0.09 & 0.12 & 0.12 & 0.00 & 0.01 & 0.01 & 0.02 \\
$\mathbf{c}_{5}$ & 1.27 & -1.23 & -0.02 & -0.04 & 0.05 & 0.10 & 0.12 & 0.13 & 0.00 & 0.01 & 0.02 & 0.02 \\
\hline
\end{tabular}


TABle 3

Parameter estimates of Das et. al. (2011) in the simulated example.

\begin{tabular}{|c|c|c|c|c|c|c|c|c|c|c|}
\hline \multirow[b]{2}{*}{$\mathrm{n}$} & \multirow[b]{2}{*}{$\sigma^{2}$} & \multirow[b]{2}{*}{ Parameters } & \multicolumn{8}{|c|}{ Estimated Legendre Coefficients } \\
\hline & & & \multicolumn{4}{|c|}{ Additive Effect } & \multicolumn{4}{|c|}{ Dominant Effect } \\
\hline \multirow[t]{5}{*}{600} & 16 & $\left(\mathbf{b}_{1}, \mathbf{c}_{1}\right)$ & 1.23 & 0.90 & -2.05 & -0.37 & 0.25 & -0.10 & 0.21 & 0.09 \\
\hline & & $\left(\mathbf{b}_{2}, \mathbf{c}_{2}\right)$ & 1.38 & -0.16 & 0.62 & -4.74 & 0.17 & -0.14 & 0.10 & 0.13 \\
\hline & & $\left(\mathbf{b}_{3}, \mathbf{c}_{3}\right)$ & 1.60 & 0.07 & -0.19 & -0.37 & 1.69 & -2.27 & 4.84 & 1.49 \\
\hline & & $\left(\mathbf{b}_{4}, \mathbf{c}_{4}\right)$ & 0.29 & 0.03 & -0.08 & -0.36 & 1.30 & 1.12 & 1.98 & 1.53 \\
\hline & & $\left(\mathbf{b}_{5}, \mathbf{c}_{5}\right)$ & 0.30 & 0.03 & -0.13 & -0.38 & 1.48 & -1.37 & 0.04 & 0.16 \\
\hline \multirow[t]{5}{*}{600} & 9 & $\left(\mathbf{b}_{1}, \mathbf{c}_{1}\right)$ & 1.29 & 0.89 & -1.97 & -0.43 & 0.20 & -0.13 & 0.19 & 0.02 \\
\hline & & $\left(\mathbf{b}_{2}, \mathbf{c}_{2}\right)$ & 1.40 & -0.15 & 0.54 & -4.73 & 0.14 & -0.09 & 0.08 & 0.07 \\
\hline & & $\left(\mathbf{b}_{3}, \mathbf{c}_{3}\right)$ & 1.58 & 0.11 & -0.15 & -0.34 & 1.62 & -2.16 & 4.89 & 1.49 \\
\hline & & $\left(\mathbf{b}_{4}, \mathbf{c}_{4}\right)$ & 0.31 & 0.06 & -0.11 & -0.39 & 1.21 & 1.21 & 1.94 & 1.56 \\
\hline & & $\left(\mathbf{b}_{5}, \mathbf{c}_{5}\right)$ & 0.33 & 0.08 & -0.13 & -0.46 & 1.44 & -1.29 & 0.13 & 0.08 \\
\hline \multirow[t]{5}{*}{600} & 4 & $\left(\mathbf{b}_{1}, \mathbf{c}_{1}\right)$ & 1.27 & 0.88 & -1.96 & -0.43 & 0.12 & -0.07 & 0.07 & 0.04 \\
\hline & & $\left(\mathbf{b}_{2}, \mathbf{c}_{2}\right)$ & 1.36 & -0.13 & 0.54 & -4.73 & 0.10 & -0.07 & 0.08 & 0.09 \\
\hline & & $\left(\mathbf{b}_{3}, \mathbf{c}_{3}\right)$ & 1.57 & 0.03 & -0.11 & -0.39 & 1.55 & -2.14 & 4.82 & 1.44 \\
\hline & & $\left(\mathbf{b}_{4}, \mathbf{c}_{4}\right)$ & 0.30 & 0.06 & -0.09 & -0.40 & 1.12 & 1.24 & 1.99 & 1.46 \\
\hline & & $\left(\mathbf{b}_{5}, \mathbf{c}_{5}\right)$ & 0.31 & 0.03 & -0.06 & -0.37 & 1.38 & -1.28 & 0.09 & 0.04 \\
\hline \multirow[t]{5}{*}{800} & 16 & $\left(\mathbf{b}_{1}, \mathbf{c}_{1}\right)$ & 1.25 & 0.85 & -2.00 & -0.40 & 0.19 & -0.15 & 0.11 & 0.11 \\
\hline & & $\left(\mathbf{b}_{2}, \mathbf{c}_{2}\right)$ & 1.36 & -0.12 & 0.59 & -4.72 & 0.16 & -0.10 & 0.04 & 0.02 \\
\hline & & $\left(\mathbf{b}_{3}, \mathbf{c}_{3}\right)$ & 1.60 & 0.07 & -0.11 & -0.42 & 1.63 & -2.21 & 4.80 & 1.42 \\
\hline & & $\left(\mathbf{b}_{4}, \mathbf{c}_{4}\right)$ & 0.30 & 0.05 & -0.09 & -0.40 & 1.17 & 1.20 & 1.97 & 1.50 \\
\hline & & $\left(\mathbf{b}_{5}, \mathbf{c}_{5}\right)$ & 0.29 & 0.03 & -0.13 & -0.39 & 1.42 & -1.28 & 0.07 & 0.06 \\
\hline \multirow[t]{5}{*}{800} & 9 & $\left(\mathbf{b}_{1}, \mathbf{c}_{1}\right)$ & 1.25 & 0.88 & -2.01 & -0.40 & 0.11 & -0.08 & 0.02 & -0.11 \\
\hline & & $\left(\mathbf{b}_{2}, \mathbf{c}_{2}\right)$ & 1.38 & -0.16 & 0.62 & -4.73 & 0.12 & -0.07 & 0.05 & 0.04 \\
\hline & & $\left(\mathbf{b}_{3}, \mathbf{c}_{3}\right)$ & 1.58 & 0.07 & -0.13 & -0.36 & 1.56 & -2.21 & 4.85 & 1.39 \\
\hline & & $\left(\mathbf{b}_{4}, \mathbf{c}_{4}\right)$ & 0.32 & 0.04 & -0.04 & -0.31 & 1.11 & 1.23 & 1.96 & 1.44 \\
\hline & & $\left(\mathbf{b}_{5}, \mathbf{c}_{5}\right)$ & 0.30 & 0.09 & -0.12 & -0.35 & 1.35 & -1.26 & 0.09 & 0.09 \\
\hline \multirow[t]{5}{*}{800} & 4 & $\left(\mathbf{b}_{1}, \mathbf{c}_{1}\right)$ & 1.26 & 0.86 & -1.99 & -0.39 & 0.09 & -0.05 & 0.06 & -0.01 \\
\hline & & $\left(\mathbf{b}_{2}, \mathbf{c}_{2}\right)$ & 1.36 & -0.15 & 0.57 & -4.71 & 0.05 & -0.03 & 0.05 & 0.05 \\
\hline & & $\left(\mathbf{b}_{3}, \mathbf{c}_{3}\right)$ & 1.60 & 0.06 & -0.09 & -0.40 & 1.55 & -2.15 & 4.79 & 1.44 \\
\hline & & $\left(\mathbf{b}_{4}, \mathbf{c}_{4}\right)$ & 0.30 & 0.06 & -0.08 & -0.40 & 1.07 & 1.27 & 1.89 & 1.56 \\
\hline & & $\left(\mathbf{b}_{5}, \mathbf{c}_{5}\right)$ & 0.28 & 0.04 & -0.10 & -0.37 & 1.34 & -1.26 & 0.05 & 0.03 \\
\hline
\end{tabular}



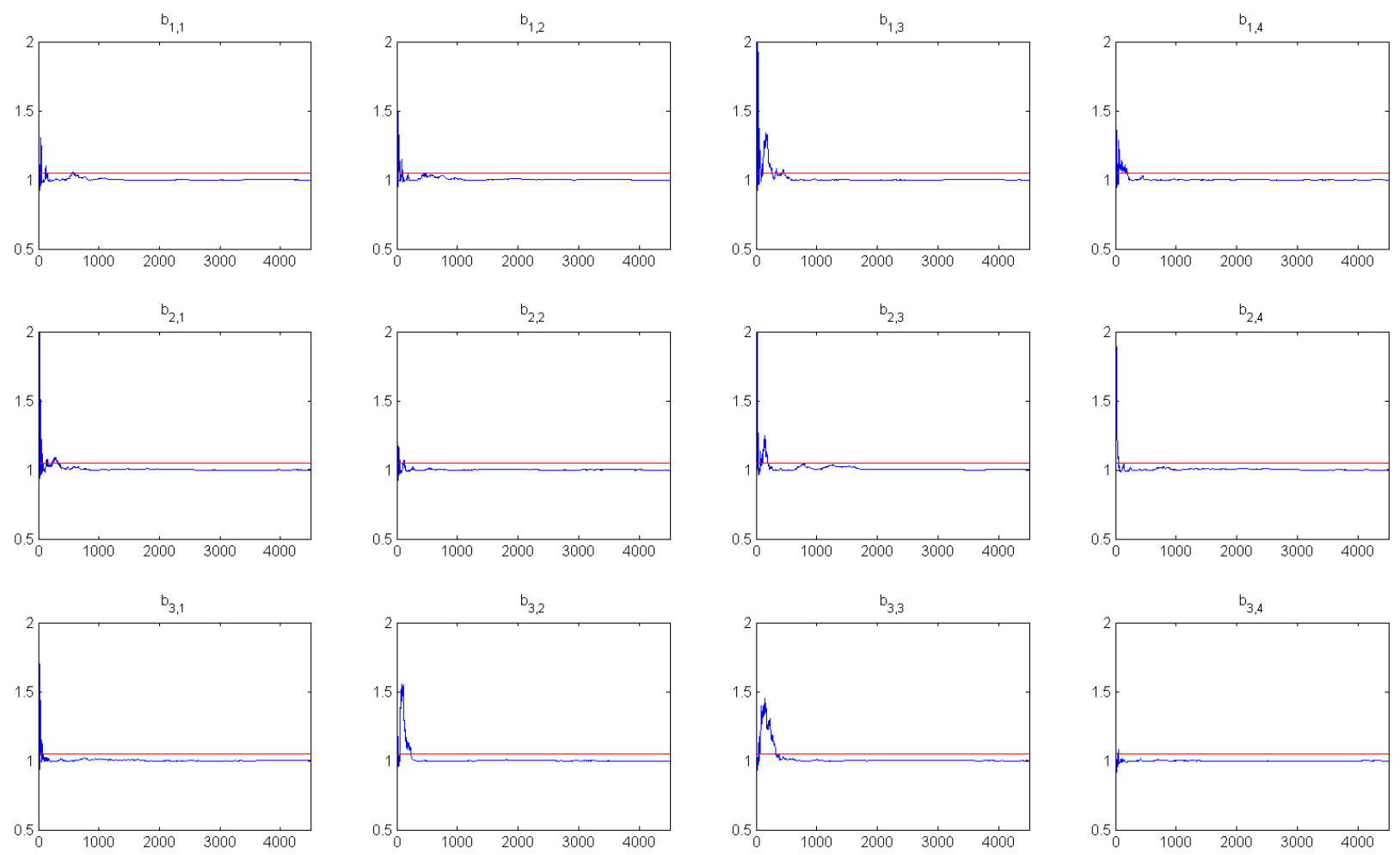

(a) PSRF for each parameter in $b_{1}, b_{2}, b_{3}$.
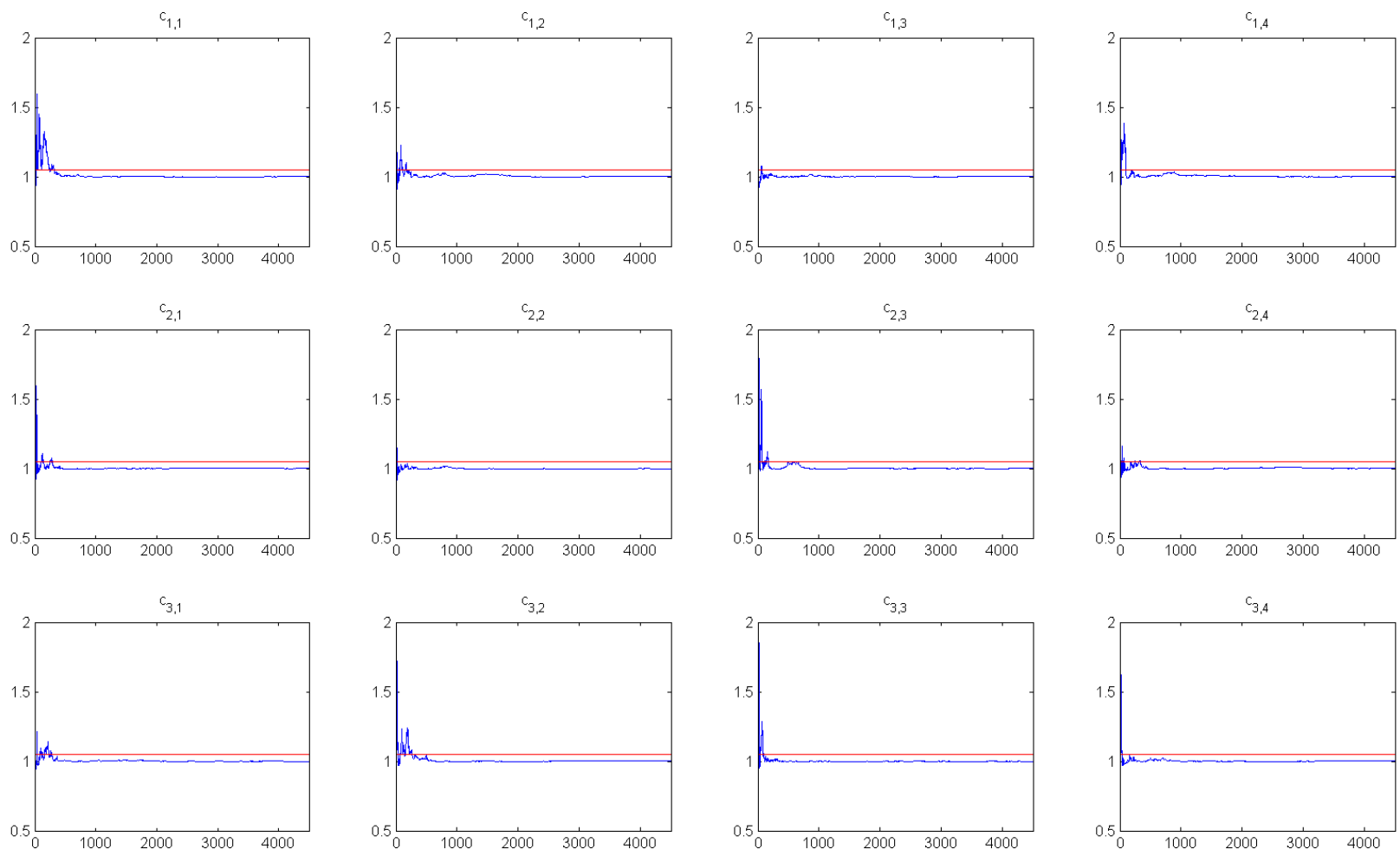

(b) PSRF for each parameter in $c_{1}, c_{2}, c_{3}$.

Fig 1: Potential scale reduction factor (PSRF) against iterations in a simulation with $n=600$ and $\sigma^{2}=16$. 